\title{
PERSPECTIVES
}

\section{Application of Game Theory to Explore the Dynamics of Host-Pathogen Association in Phytobiomes}

\author{
Man S. Kim, Huan Zhang, and Won Bo Shim, ${ }^{\dagger}$ Department of Plant Pathology and Microbiology, Texas A\&M \\ University, College Station 77843-2132
}

Accepted for publication 27 July 2018.

\begin{tabular}{ll} 
ABSTRACT \\
$\begin{array}{ll}\text { Recent advances in sequencing technology and big data analytics } & \begin{array}{l}\text { conceptualize the complex nature of associations that occur between } \\
\text { plants and pathogens. However, equipped with vastly improved }\end{array} \\
\text { are moving plant pathology into a new era where we are now } & \text { technology we now have opportunities to further explore } \\
\text { pursuing a deeper systems-level understanding of host-pathogen } & \text { multicomponent host-pathogen associations as well as } \\
\text { associations. For past several decades, scientists have } & \text { microbe-microbe associations in phytobiomes and agroecosystems. } \\
\text { predominantly tried to delineate and streamline these interactions } \\
\text { into single pathogen-single host model systems, using reductionist } \\
\begin{array}{l}\text { experimental approaches. As many of us are aware, there have been } \\
\text { several significant proposed ideas and models to help simplify and }\end{array}\end{array} \quad \begin{array}{l}\text { concepts could be adopted to study the dynamics of complex } \\
\text { phytobiomes and to help us gain fresh and transformative insight. }\end{array}$ \\
\hline
\end{tabular}

Applying statistical and computational analyses to gain deeper insight into plant-pathogen associations is nothing new, as demonstrated by countless research and review articles published in recent years. We cannot revisit all these publications, and obviously that is beyond the scope of our article. But here we would like to briefly discuss three recent reviews that provide some conceptual background. First, a review article by Pritchard and Birch (2011) thoroughly discussed how systems-level interpretation through network modeling of pathogen effectors and host receptors can lead to innovative discoveries in plant-microbe interactions. This review also clarified a number of intracellular and intercellular network biology concepts, including scale-free network, hub, power law, robustness, and topology. But the authors also shared technical and logistical challenges, including the dynamic nature of interactions and degree of correlation among datasets. Notably, Pritchard and Birch (2011) stipulated that among all 'omics" technologies, transcriptomics is perhaps the most recognizable and pervasive tool currently available to plant pathologists when trying to gain insight

\section{†Corresponding author: W. B. Shim; E-mail: wbshim@tamu.edu}

Author contributions: Research concepts performed by M.K., H.Z., and W.B.S.; experiments performed by M.K. and H.Z.; and manuscript preparation performed by M.K. and W.B.S. All authors reviewed and approved the manuscript.

Funding: This work was supported by the U.S. Department of AgricultureAgriculture and Food Research Initiative grant 2013-68004-20359. into dynamic host-microbe associations. Next, Schenk et al. (2012) raised the question whether transcriptomics, particularly the multispecies transcriptomics (similar concepts are also known as parallel transcriptomics, metatranscriptomics, or interaction transcriptomics), can help improve our understanding of complex interactions between the host and microbes in agroecosystem. Not surprisingly, since the publication the technology has vastly improved and become more affordable, and perhaps tens of thousands of sequencing tasks since have been performed. While valuable information has been gained in many host-microbial systems, majority of these sequencing efforts have led to descriptive summarizations of massive datasets and fell short in exploring deeper into the systems-level understanding that Pritchard and Birch (2011) discussed. Very recently, Abdullah et al. (2017) made a case for multicomponent investigatory approach in host-pathogen interactions, not simply laying out tools but providing conceptual description of recent research efforts and events. The authors scrutinized limitations associated with simplified models, reductionist research methods, and outcome-driven observations focusing on single microbe-single host systems, and emphasized the need to focus on investigating the dynamics of host-multipathogen associations. The important question now is how?

The daunting challenge lies in the experimental design and analytical strategy that will allow us to gain insight into the dynamic multicomponent interactions between the host and a complex mixture of microbes. Unfortunately, there is no straightforward solution currently available. Our laboratory has been studying maize-Fusarium verticillioides association and recently initiated transcriptome analyses of this pathosystem (Kim et al. 2018a, b). As 
we struggled to develop computational strategies to analyze our datasets, we could not help but ask the question; are there any theoretical foundation for developing an approach that can help us investigate the dynamics of multipathogen-host associations. Pritchard and Birch (2014) recently argued that many of the current expository models show limitations in providing insight into multiple components of dynamic systems. We felt the need to explore a new predictive strategy that can generate better understanding of our pathosystem from raw datasets. Many plant pathologists frequently refer to host-pathogen interaction as a warfare. However, a warfare is anything but a series of sequential, linear host and/or pathogen reactions that triggers biochemical signals. Warfare is composed of a complex collection of multifaceted, multidimensional, incessant exploits by players, and this is very similar to those actions anticipated in multipathogen-host associations, e.g., antagonism, synergism, deception, cooperation, or aggression. We need a fresh perspective to gain new and transformative insight into complex phytobiomes, and here we are proposing to adopt game theory concepts to study the dynamics of multipathogen-host associations.

Game theory is the study of mathematical models of conflict, negotiation and cooperation between decision-makers (also known as "players") (Davis 1983). In its applications, however, the scope of game theory is vastly greater than what would be expected from the fundamental methodology itself (Fisher 2008). It has mainly offered insights into problems not only in economics, psychology, and political science, but also even in computer science and biology (Harsanyi and Selten 1988; Myerson 2013; Osborne and Rubinstein 1994). In biology, evolutionary game theory (EGT) as a mathematical model has become one of the major theoretical approaches in evolutionary biology, population biology, and ecology studies since this view was introduced in animal conflicts (Nowak and Sigmund 2004; Smith and Price 1973). EGT has been also applied to cancer biology when investigating complicated mechanism between cells as players to study the long-term proliferation of cancer cells (Archetti 2013; Basanta et al. 2012; Kianercy et al. 2014). In plant ecology, the contribution of EGT can be found in numerous conceptual tools used in research. One example is how the concept of evolutionary stable strategy (ESS) is used to explain different evolutionary trajectories, mainly how a community can resist challenges from individuals with any other strategy (Apaloo et al. 2009). A recent review by McNickle and Dybzinski (2013) explains how plant traits can be thought of as its strategies that can be adapted to neighboring plants' strategies and how biological market theory can help expand viewpoints and predictions in plant ecology research. In host-microbe association studies game theory is also making an impact, where researchers are using scenarios such as prisoner's dilemma and collective investment game to interpret how multiple organisms strategically utilize their tools to cooperate and compete to gain fitness advantage (Huang et al. 2007; Madgwick et al. 2018; Mukherjee et al. 2013; Turner and Chao 1999). However, we have seen limited examples of game theory-like interpretations applied to plant-pathogen interactions and phytobiomes research (Abdullah et al. 2017; Mommer et al. 2018; Tsunoda and van Dam 2017). We have not yet encountered the application of game theory to interpret comprehensive plant-pathogen associations at the molecular level.

In the disease triangle (Fig. 1A), each component has single linear association with other components. However, this model has limitations when trying to understand multicomponent associations in agroecosystems. Here, we are proposing a multipathogen-host association model in an effort to comprehensively consider interactions between hosts and multiple pathogens during

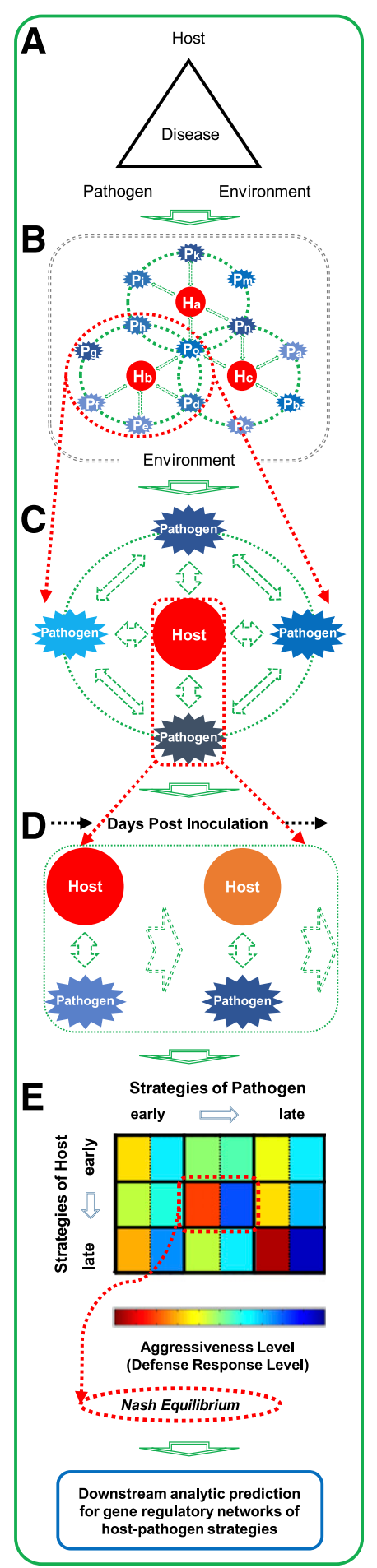

Fig. 1. Proposed application of game theory model to multipathogen-multihost phytobiomes. A, Disease triangle model. B, Disease triangle conceptually expanded to consider phytobiomes (agroecosystem), where multiple hosts and pathogens dynamically interact with each other. ' $\mathrm{H}$ ' and ' $\mathrm{P}$ ' stand for a host and a pathogen, respectively. $\mathrm{Ha}, \mathrm{Hb}$, and $\mathrm{Hc}$ represent different hosts. C, Host-multipathogen optimization. We can arbitrarily setup host-multipathogen association for research purpose. D, Dynamic modulation of multicomponent association, with possible scenario of time-course analysis for host-pathogen interaction. E, Equilibria identified by appropriately modified game theory-based optimization. This is an example of game theory based approach for time-course analysis to identify optimized states (i.e., Nash equilibrium) by considering aggressiveness and defense response levels as time progress. 
pathogenesis in the field (Fig. 1B to E). The underlying hypothesis of this model is that a multipathogen-host association is a battle between a defender (host) and multiple invaders (pathogens) with countless dynamic strategies and tools employed between these players, even among pathogens. Our proposed analytical strategy conceptually takes into account diverse pathogen strategies they utilize to attack their host. Table 1 provides a brief explanation of terminology comparison between original game theory and our proposed application in host-pathogen associations. The model considers that certain host can have restricted interaction with an individual pathogen, whereas others can be exposed to a broad range of pathogens. For example, in Figure 1B, some pathogens, e.g., Pi or $\mathrm{Pk}$, target a specific single host, e.g., Ha, while other pathogens, e.g., Po, can attack multiple hosts, e.g., Ha, Hb, and Hc. From Figure 1B, we can preferentially take into consideration a typical case, multipathogen-host (Fig. 1C), since pathogens in the field are generally postulated to attack their host cooperatively or selectively using numerous adaptive, self-optimized strategies in a complicated manner. Also, we must consider disease development in a timecourse manner in host-pathogen associations and investigate how they dynamically modulate their strategies over time, even under variable environmental conditions. For instance (Fig. 1D), it can be hypothesized that simultaneous games are continuously played, among players (host or pathogens) with various strategies during disease progression. At each time point, we can anticipate that a player will display its maximum virulence/defense aptitude through deploying its most effective strategy while recognizing the optimum strategies of other players. The maximum levels of virulence and resistance used as the matrix inputs (Fig. 1E) can be estimated by collectively considering expression levels of genes associated with virulence and resistance, respectively. In game theory matrix (Fig. 2), optimum or suboptimum states (pure strategy Nash equilibrium or mixed strategy Nash equilibrium), where every player exhibits its best, functional response, can be identified (Fisher 2008). And this will allow us to select the state where downstream analysis (i.e., to identify critical gene regulatory networks) can be subsequently performed. To factor in such parameters from multicomponent and multilevel perspectives in quantitative biological research, we are proposing an analytical approach utilizing game theory-based optimization along with

TABLE 1

Comparison between basic game theory and our proposed phytobiome application (simultaneous games with complete information)

\begin{tabular}{lll}
\hline Key concepts & In original game theory & In phytobiome application \\
\hline Decision makers (players) & Humans & Plants and pathogens \\
\hline Payoffs & Rewards (financial and/or emotional) & Survival and/or reproduction \\
\hline Goal & Maximize payoffs & Maximize payoffs \\
\hline Decision making & Human instinct (greed or selfishness) & Innate instinct for survival \\
\hline Game scenarios & $\begin{array}{l}\text { Finite number of strategies in a given game } \\
\text { condition }\end{array}$ & $\begin{array}{c}\text { Finite number of strategies at a given time point but can } \\
\text { be infinite and dynamic during pathogenesis }\end{array}$ \\
\hline Communication & Only through their strategies & $\begin{array}{c}\text { A host intrinsically communicates only with specific } \\
\text { pathogens, or certain number of pathogens }\end{array}$ \\
\hline Underlying assumption on strategies & $\begin{array}{c}\text { Each player is aware of strategies and payoffs of } \\
\text { the other players }\end{array}$ & $\begin{array}{c}\text { A player (host or pathogen) inherently knows how } \\
\text { strong the others are and what strategies the others } \\
\text { have }\end{array}$ \\
\hline
\end{tabular}

A

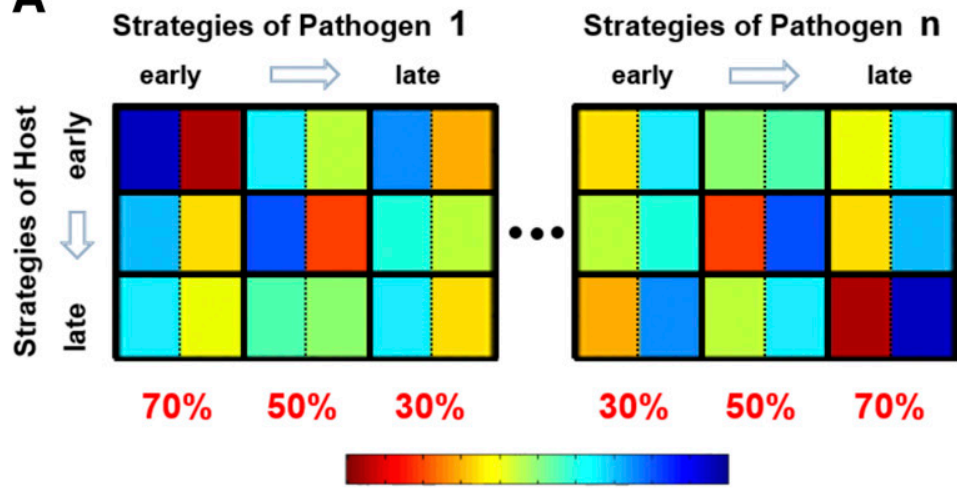

Aggressiveness Level (Defense Response Level)
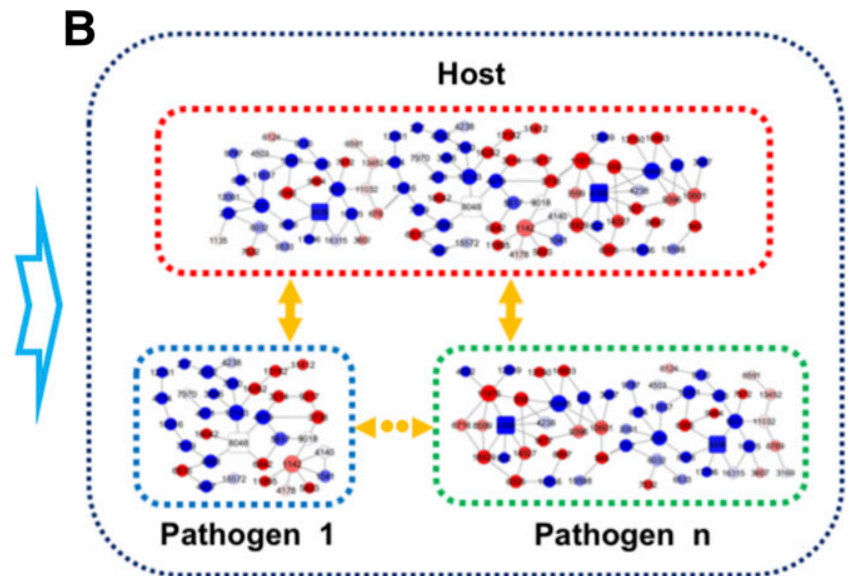

Fig. 2. An example of host-multipathogen interaction analysis using Bayesian game-based gene regulatory network optimization. Quantitative transcriptome profiling data can be used to predict virulence level or resistance level, thus giving us when and how each pathogen is dominant over other pathogens against the host. A, Pathogen 1 may not have complete information (game with imperfect information) about pathogen $\mathrm{n}$ in a real biological environment. However, pathogen 1 can have "beliefs" with known probability distribution about pathogen n's strategies, and against the host. Using this probability distribution (Bayesian game theory), we can infer relative influence between players (i.e., expected virulence level or defense level). B, Subsequently, once we define Nash equilibrium state, network-based sequence analysis pipeline can be applied to predict gene regulatory networks (i.e., at transcriptome level) in multiple host-pathogen associations. 
our previously developed computational network biology analysis (Kim et al. 2015, 2018a, b). This approach will allow us to develop a systematic time-dependent network-based pipeline to identify gene regulatory networks of strategies from both host and pathogen in various plant microbiome conditions. In this pipeline, equilibria, e.g., pure strategy Nash equilibrium or mixed strategy Nash equilibrium, of 'omic' data identified by appropriately modified game theory-based optimization will be target

A

B

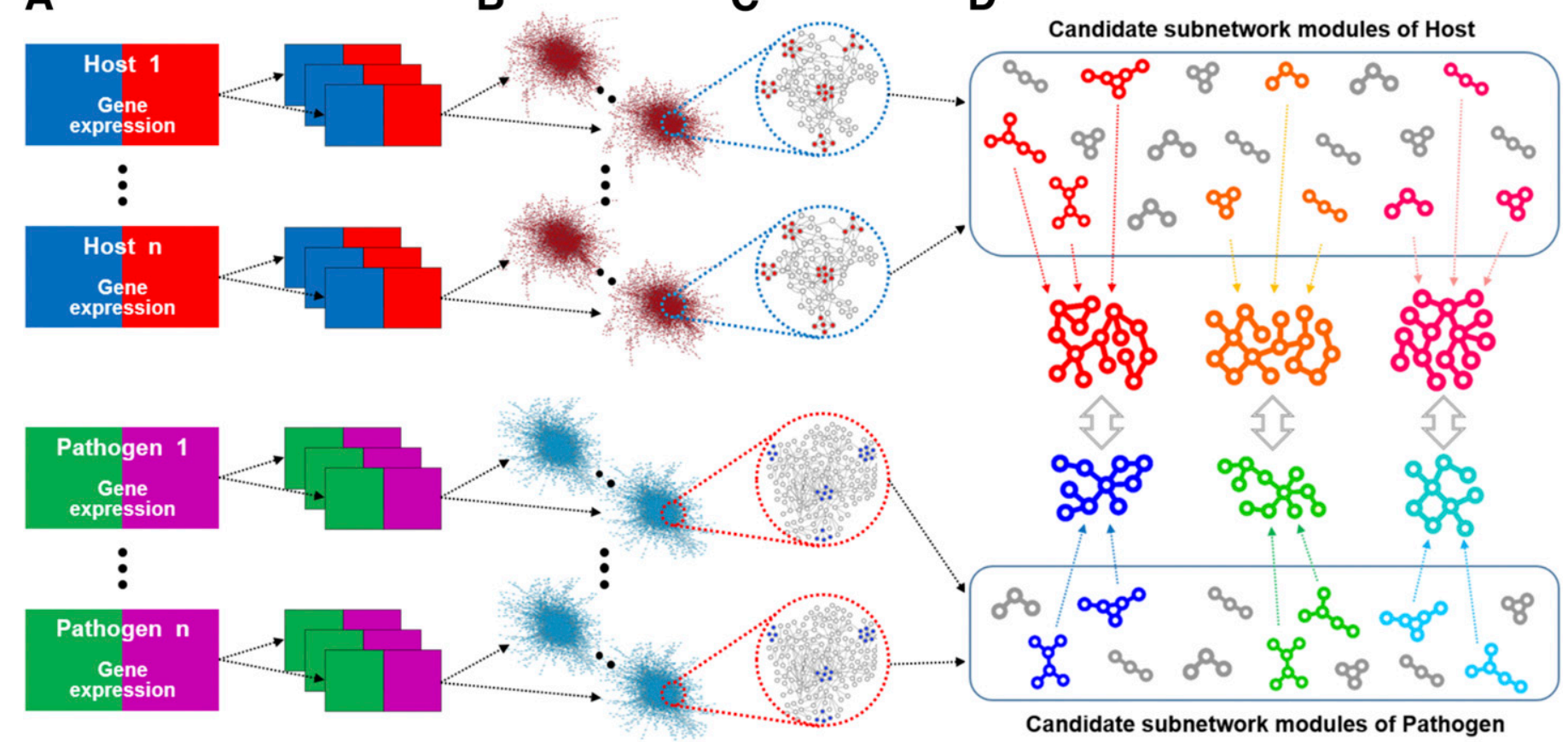
C D

D Candidate subnetwork modules of Host

Fig. 3. An overview of potential subnetwork module inference procedure in multiple host-pathogen association. After selecting Nash equilibrium states through the game theory-based optimization, we can continue to our potential subnetwork module inference approach to identify gene regulatory mechanism. A, Process transcriptome data through different combinations of normalization methods. B, Infer multilayered co-expression networks through various approaches by considering uncertainty in biological data with multiple threshold cut-offs. C, Predict subnetwork modules on the diversely built co-expression networks for players (such as hosts and pathogens). D, Link subnetwork modules from each player against others if they associate in competition and/or cooperation.

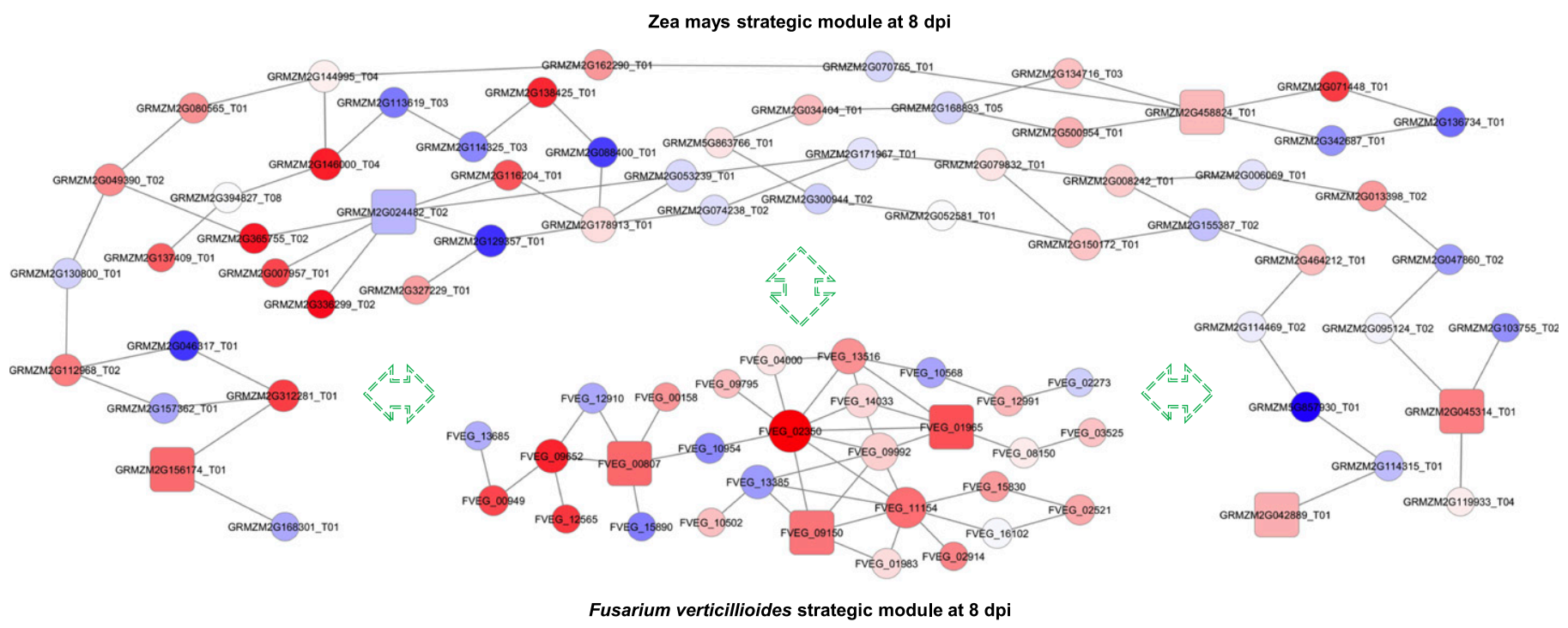

higher expression in 8 dpi than $6 \mathrm{dpi}$

lower expression in 8 dpi than 6 dpi

Fig. 4. Proposed game theory-based strategic gene regulatory network prediction between maize-Fusarium verticillioides at 8 days postinoculation (dpi). Here, specific strategic modules between maize and $F$. verticillioides are shown. These strategic modules come from the maize- $F$. verticillioides interaction process between 6 and $8 \mathrm{dpi}$. In this figure, each node represents a gene of either $F$. verticillioides or Zea mays. Edges are inferred by considering strong correlation between genes. Each node size corresponds with number of edges that the node contains. The color is proportional to degree of differentiation, where upregulation on 8 or 6 dpi indicates red or blue, respectively. 
stages (i.e., time points of interest), where strategic functional subnetwork modules of components from pathogens and hosts can be analyzed for virulence and defense responses, respectively. For example, a Nash equilibrium for host-pathogen association would indicate that all pathogens and the host are simultaneously performing their optimal functional responses against those actions of others. When trying to comprehend this concept from the host viewpoint, we can hypothesize that the host will be maximizing its defense strategy to minimize the damage stemming from diverse attacks from pathogens when pathogens themselves will concurrently be seeking their own advantages against each other (Fig. 2). While coping with the damage inflicted by pathogens, the host will agree to an optimized state (i.e., Nash equilibrium) since the host would inherently recognize that altering its defense strategy cannot lessen the damage any further.

Once we select Nash equilibrium states through the game theorybased optimization, we can continue to our potential subnetwork module inference approach to identify gene regulatory mechanism by following steps depicted in Figure 3. Transcriptome data are first processed, and multilayered co-expression networks can be inferred through various approaches by considering uncertainty in biological data with multiple threshold cut-offs. Next, subnetwork modules can be predicted on the diversely built co-expression networks for players (hosts and pathogens), and combined on each side if they associate amicably with other modules. Lastly, subnetwork modules from each player against others can be linked if they associate in competition and/or cooperation. A possible game theory-based strategic gene regulatory network prediction of host-pathogen association, where significantly and differentially activated multiple subnetwork modules combined for either cooperation (in host or pathogen) or competition (between host and pathogen), is depicted in Figure 4 using maize- $F$. verticillioides system. In this exploratory analysis attempt, we performed our proposed approach to identify either pathogen virulence or host defense functional modules at the 8-days postinoculation time point, our optimized pure strategy Nash equilibrium. As illustrated in Figure 4, for the potential module of virulence strategy in $F$. verticillioides, three known ear rot virulence genes, such as FvMCM1 (FVEG_01965), FvHAP2 (FVEG_00807), and SGE1 (FVEG_09150), were significantly activated by not only sharing strong correlation with each other but also other subnetwork member genes. For the potential module of maize defensive strategy, six maize genes (GRMZM2G042889_T01, GRMZM2G458824_ T01, GRMZM2G024482_T02, GRMZM2G156174_T01, and GRMZM2G045314_T01) with annotated GO term GO:0009814 (defense response to fungus, incompatible interaction) showed vigorous activation with other maize subnetwork member genes in a coordinated manner. This example demonstrates how analyses of group of gene subnetworks can help understand system-wide host-pathogen association, and perhaps further assembled to reveal plant microbiome equilibria.

We recognize the limitations and potential pitfalls of our proposed approach since no single method can unambiguously unveil the most optimized and efficient strategy underlying gene regulatory mechanism for plant microbiome. We first and foremost acknowledge that the classical game theory scenarios are mainly based on human characteristics, such as self-interest, cooperation, sacrifice, and therefore specific theories that cater to human behaviors, i.e., prisoner's dilemma, chicken, and volunteer's dilemma, may not be readily applicable to plant-pathogen associations. Also, as the number of participants (hosts or pathogens) increase, analyzing exponentially growing datasets and the combination of mixed strategies in game theory can be immensely challenging computationally and logistically. Finally, uncertainty triggered by unexpected factors (i.e., abiotic factors) in agroecosystems can pose difficulties. The key aim of this article is to facilitate dialogues in our field about how current phytobiomes research is predominantly focused on pattern description but is very slow in moving toward a predictive stage. Observational data can provide distribution and correlation analyses (e.g., principal component analysis), but can be limited in providing predictive insights into the dynamic multicomponent associations between plants and the complex community of microbes. Our proposed strategy may not completely explain underlying principles of association between host and pathogens, but it can lead to fresh and novel interpretation of "games" played between multiple organisms in phytobiomes and agroecosystem.

Availability of materials and data. The datasets analyzed during the current study are available from the corresponding author on reasonable request.

\section{LITERATURE CITED}

Abdullah, A. S., Moffat, C. S., Lopez-Ruiz, F. J., Gibberd, M. R., Hamblin, J., and Zerihun, A. 2017. Host-multi-pathogen warfare: Pathogen interactions in co-infected plants. Front. Plant Sci. 8:1806.

Apaloo, J., Brown, J. S., and Vincent, T. L. 2009. Evolutionary game theory: ESS, convergence stability, and NIS. Evol. Ecol. Res. 11:489-515.

Archetti, M. 2013. Evolutionary game theory of growth factor production: Implications for tumour heterogeneity and resistance to therapies. Br. J. Cancer 109:1056-1062.

Basanta, D., Scott, J. G., Fishman, M. N., Ayala, G., Hayward, S. W., and Anderson, A. R. A. 2012. Investigating prostate cancer tumour-stroma interactions: Clinical and biological insights from an evolutionary game. Br. J. Cancer 106:174-181.

Davis, M. D. 1983. Game Theory: A Nontechnical Introduction. Dover Publications, Inc., Mineola, New York.

Fisher, L. 2008. Rock, Paper, Scissors: Game Theory in Everyday Life. Basic Books, New York.

Harsanyi, J. C., and Selten, R. 1988. A General Theory of Equilibrium Selection in Games. MIT Press, Cambridge, MA.

Huang, S. H., Zhou, W. S., Jong, A., and Qi, H. 2007. Game theory models for infectious diseases. Pages 265-269 in: Proceedings of the Frontiers in the Convergence of Bioscience and Information Technologies. The Institute of Electrical and Electronic Engineers, Jeju, Korea.

Kianercy, A., Veltri, R., and Pienta, K. J. 2014. Critical transitions in a game theoretic model of tumour metabolism. Interface Focus 4: 20140014.

Kim, M., Zhang, H., Woloshuk, C., Shim, W. B., and Yoon, B. J. 2015. Computational identification of genetic subnetwork modules associated with maize defense response to Fusarium verticillioides. BMC Bioinformatics 16: S12.

Kim, M., Zhang, H., Woloshuk, C. P., Shim, W. B., and Yoon, B. J. 2018b. Computational prediction of pathogenic network modules in Fusarium verticillioides. IEEE/ACM Trans. Comput. Biol. Bioinformatics 15: 506-515.

Kim, M., Zhang, H., Yan, H., Yoon, B. J., and Shim, W. B. 2018a. Characterizing co-expression networks underpinning maize stalk rot virulence in Fusarium verticillioides through computational subnetwork module analyses. Sci. Rep. 8:8310.

Madgwick, P. G., Stewart, B., Belcher, L. J., Thompson, C. R. L., and Wolf, J. B. 2018. Strategic investment explains patterns of cooperation and cheating in a microbe. Proc. Natl. Acad. Sci. USA 115:E4823-E4832.

McNickle, G. G., and Dybzinski, R. 2013. Game theory and plant ecology. Ecol. Lett. 16:545-555.

Mommer, L., Cotton, T. E. A., Raaijmakers, J. M., Termorshuizen, A. J., van Ruijven, J., Hendriks, M., van Rijssel, S. Q., van de Mortel, J. E., van der Paauw, J. W., Schijlen, E. G. W. M., Smit-Tiekstra, A. E., Berendse, F., de Kroon, H., and Dumbrell, A. J. 2018. Lost in diversity: The interactions between soil-borne fungi, biodiversity and plant productivity. New Phytol. 218:542-553.

Mukherjee, S., Sambarey, A., Prashanthi, K., and Chandra, N. 2013. Current trends in modeling host-pathogen interactions. Wires Data Mining Knowl. Discov. 3:109-128. 
Myerson, R. B. 2013. Game Theory. Harvard University Press, Cambridge, MA.

Nowak, M. A., and Sigmund, K. 2004. Evolutionary dynamics of biological games. Science 303:793-799.

Osborne, M. J., and Rubinstein, R. 1994. A Course in Game Theory. MIT Press, Cambridge, MA.

Pritchard, L., and Birch, P. 2011. A systems biology perspective on plantmicrobe interactions: Biochemical and structural targets of pathogen effectors. Plant Sci. 180:584-603.
Pritchard, L., and Birch, P. R. J. 2014. The zigzag model of plant-microbe interactions: Is it time to move on? Mol. Plant Pathol. 15:865-870.

Schenk, P. M., Carvalhais, L. C., and Kazan, K. 2012. Unraveling plant-microbe interactions: Can multi-species transcriptomics help? Trends Biotechnol. 30:177-184.

Smith, J. M., and Price, G. R. 1973. Logic of animal conflict. Nature 246:15-18.

Tsunoda, T., and van Dam, N. M. 2017. Root chemical traits and their roles in belowground biotic interactions. Pedobiologia (Jena) 65:58-67.

Turner, P. E., and Chao, L. 1999. Prisoner's dilemma in an RNA virus. Nature 398:441-443. 